\title{
Radius-Dependent Inhibition and Activation of Chemical Oscillations in Small Droplets
}

\author{
Oliver Steinbock* and Stefan C. Muiller \\ Otto-von-Guericke-Universität Magdeburg, Institut für Experimentelle Physik, Abteilung Biophysik, \\ Universitätsplatz, 2, D-39106 Magdeburg, Germany
}

Received: March 10, 1998; In Final Form: June 1, 1998

\begin{abstract}
The oscillatory dynamics in droplets of the ferroin-catalyzed Belousov-Zhabotinsky solution is investigated. Due to a diffusive influx of molecular oxygen from a surrounding hydrophobic phase, droplets experience an increase in oscillation period with decreasing drop radius. If the radius is smaller than approximately 1.5 $\mathrm{mm}$, no oscillations are observed. The phase of oscillations showed a radial dependence that gives rise to nontransient phase waves propagating from the center outward. In the absence of oxygen, the period decreases slightly with decreasing drop radii. A simple geometrical analysis qualitatively explains the main experimental observations. The analysis stems from an approach that relates the efficiency of inhibition to the surfaceto-volume ratio of droplets.
\end{abstract}

\section{Introduction}

The surface-to-volume ratio is an important parameter in determining the design of living species, since it controls the exchange of information and matter between environment and organism. ${ }^{1,2}$ An increase in size, for example, could decrease the oxygen supply and hinder an efficient release of $\mathrm{CO}_{2}$ unless the surface area increases allometrically to maintain the surface/ volume ratio. ${ }^{2}$ In this paper, we present results on the effect of the surface-to-volume ratio on oscillations in the BelousovZhabotinsky (BZ) reaction. ${ }^{3}$ The BZ reaction is a well-known example for self-organizing chemical systems far from equilibrium and is often considered to be a chemical model for certain biological media. ${ }^{4}$ Spatially homogeneous BZ systems show a variety of phenomena such as nonlinear oscillations, period doubling, or deterministic chaos. ${ }^{5,6}$ In spatially extended reaction media traveling waves of excitation ${ }^{7}$ can be observed that have fascinating analogies to $\mathrm{Ca}^{2+}$ waves in oocytes ${ }^{8}$ or cAMP waves observed during the aggregation of slime molds. ${ }^{9}$ These analogies arise from the underlying reaction-diffusion mechanisms that couple nonlinear local kinetics by short-range diffusion. ${ }^{10}$

The BZ reaction involves the oxidation of malonic acid by an acidified bromate solution in the presence of metal ion catalysts (e.g., $\mathrm{Ce}(\mathrm{III}) / \mathrm{Ce}(\mathrm{IV})$ or ferroin/ferriin). The intermediate $\mathrm{HBrO}_{2}$ acts as the autocatalytic species, and its diffusion enables wave propagation. After autocatalysis has ceased, the system is in a refractory state characterized by high concentrations of the inhibitor bromide. During this phase bromine is formed according to the equilibrium ${ }^{3,5}$

$$
\mathrm{Br}^{-}+\mathrm{HOBr}+\mathrm{H}^{+} \leftrightarrow \mathrm{Br}_{2}+\mathrm{H}_{2} \mathrm{O}
$$

The redox catalyst experiences two major reactions: (a) oxidation due to $\mathrm{BrO}_{2}{ }^{-}$that is rapidly formed during the autocatalytic excitation phase and (b) reduction due to the reaction with malonic acid and its derivatives. ${ }^{3}$

Oscillations and wave propagation in the $\mathrm{BZ}$ reaction are strongly affected by molecular oxygen. ${ }^{11}$ While oxygen seems

* To whom correspondence should be addressed. to have no effect on the inorganic subset of the $\mathrm{BZ}$ reaction, ${ }^{12}$ it can react with malonyl and/or bromomalonyl radicals, causing an increased production of the inhibitor bromide. ${ }^{13,14}$ Although the malonyl ${ }^{13}$ and the peroxymalonyl ${ }^{14}$ radical have been characterized by means of EPR spectroscopy, little is known on the kinetics of radicals produced in the oxidation of bromomalonic acid by metal ions and metal ion complexes. Krug et al. ${ }^{15}$ suggested the following reaction scheme to account for an increased production of bromide under aerobic reaction conditions:

$$
\begin{gathered}
\mathrm{R}^{\bullet}+\mathrm{O}_{2} \rightarrow \mathrm{ROO}^{\bullet} \\
\mathrm{ROO}^{\bullet}+\mathrm{BrMA}^{\rightarrow} \mathrm{BrMA}^{\bullet}+\mathrm{ROOH} \\
\mathrm{BrMA}^{\bullet}+\mathrm{H}_{2} \mathrm{O} \rightarrow \mathrm{HOC}^{\bullet}(\mathrm{COOH})_{2}+\mathrm{H}^{+}+\mathrm{Br}^{-}
\end{gathered}
$$

where $\mathrm{R}^{\bullet}$ is a species not explicitly assigned to the malonyl radical, since inhibiting effects of oxygen are also observed in reduced ferroin-catalyzed BZ systems. ${ }^{16}$ For the Ce(IV)catalyzed reaction, however, $\mathrm{R}^{\bullet}$ should be identical to the malonyl radical. Alternatively to reaction R4, Försterling et al. ${ }^{17}$ suggested the reactions

$$
\begin{aligned}
& 2 \mathrm{BrMA}^{\bullet}+\mathrm{H}_{2} \mathrm{O} \rightarrow \mathrm{CBrOH}(\mathrm{COOH})_{2}+\mathrm{BrMA}^{-} \\
& \mathrm{CBrOH}(\mathrm{COOH})_{2} \rightarrow \mathrm{OC}(\mathrm{COOH})_{2}+\mathrm{H}^{+}+\mathrm{Br}^{-}
\end{aligned}
$$

The clarification of these mechanistic questions requires further experimentation. Nevertheless, different approaches have been suggested to simulate oxygen effects in the framework of the Oregonator model. Krug et al. added a constant source of bromide production to the Oregonator that solely depends on the oxygen concentration. ${ }^{15}$ Zhabotinsky et al. modified an Oregonator-type model where oxygen inhibition is expressed by an oxygen dependence of the stoichiometric factor $f$ that relates $\mathrm{Br}^{-}$production to the reduction of the catalyst. ${ }^{18}$ While the first approach denies any correlation between oxygen inhibition and the concentration of the oxidized catalyst, the latter one disregards the possibility of oxygen-induced bromide 
production in the reduced system. Note that both approaches do not explicitely consider the consumption of oxygen.

The aim of this paper is (a) to present additional data on the aspects of oxygen inhibition that should provide an interesting test case for future modeling of the aerobic BZ reaction and (b) to investigate the phenomena arising from a diffusive exchange of compounds between aqueous BZ droplets with hydrophobic surroundings. The observed phenomena are closely related to the surface-to-volume ratio of the droplets and can be qualitatively described on the basis of geometrical considerations.

\section{Experimental Section}

Small drops of BZ solution were pipetted into a glass Petri dish (diameter $9 \mathrm{~cm}$ ) containing $50 \mathrm{~mL}$ of a chemically inert silicone oil. The droplets sank to the bottom of the dish within less than a minute. Once in contact with the glass the drops flattened immediately. The resulting droplets had a circular base with radii being in the range $0.1-6.0 \mathrm{~mm}$. (Larger drops tend to show deviations from a circular base.) The initial reagent concentrations of the $\mathrm{BZ}$ solution were $\left[\mathrm{NaBrO}_{3}\right]=0.2 \mathrm{M}$, $\left[\mathrm{CH}_{2}(\mathrm{COOH})_{2}\right]=0.4 \mathrm{M}$, and [ferroin] $=4.0 \mathrm{mM}$. The concentration of $\mathrm{H}_{2} \mathrm{SO}_{4}$ was varied between 0.1 and $0.14 \mathrm{M}$. All chemicals were of analytical grade. Temperature was kept constant at $T=25.0 \pm 0.5^{\circ} \mathrm{C}$. For experiments under anaerobic conditions, the oil was purged with nitrogen gas prior to the experiment (purging time: at least $45 \mathrm{~min}$ ). Oscillations were detected using a CCD video camera (Hamamatsu C2400) monitoring the reflection of diffuse light at a wavelength of $490 \mathrm{~nm}$. An analogue image processor (Hamamatsu Argus 20; C3930-50) was employed to enhance video signals that were then digitized by a PC-based image aquisition board (Hamamatsu M4414).

\section{Experimental Results}

Figure 1 shows a typical top view of several BZ droplets. There is a large difference between the height of the drops and the radius of their base. Accordingly, the contact angle between the aqueous BZ phase and the glass surface is small $\left(\varphi \approx 10^{\circ}\right)$. Under the experimental conditions specified above this angle showed no temporal variations. ${ }^{19}$ The concentrations of the used BZ solutions gave rise to an oscillatory behavior of the medium. The main phenomena presented in the following do also occur when the drops are in direct contact to the air (or nitrogen atmosphere), but evaporation of droplets would have hindered systematic investigations significantly. In our experiments, the distance between different drops was typically $0.3-1 \mathrm{~cm}$. However, no evidence for coupling between neighboring drops was found that could have given rise to any synchronization effects.

Image sequences showing the system in its top view were analyzed by integrating the intensity signals of all pixels located within single droplets. These average signals were recorded for four oscillations starting immediately after completion of the preparation. Oscillation periods were extracted from these data. Under aerobic reaction conditions a pronounced dependence between the period $T$ of chemical oscillations and the drop radius $R$ was observed (Figure 2a). The period of BZ droplets having a radius of about $1.5 \mathrm{~mm}$ is approximately $75 \%$ higher than the period found for drop radii above $4 \mathrm{~mm}$. Below $r=1.5 \mathrm{~mm}$ no oscillations were detected within the duration of the experiment ( $>45 \mathrm{~min})$. It should be noted that the signals obtained from the oscillating drops had an excellent signal-tonoise ratio down to the smallest radii investigated. The droplets

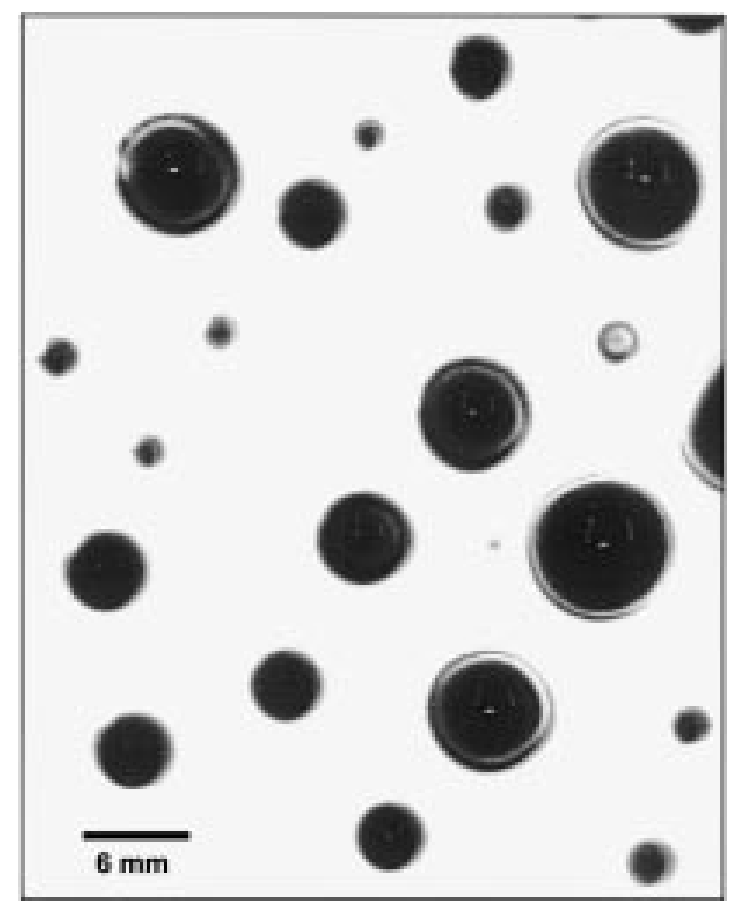

Figure 1. Several flat droplets of an oscillating BZ solution shown from a top view. Droplets are covered with an inert oil. Some of the dark drops (reduced state) show bright rings which correspond to locally excited regions (oxidized state). The oscillation period depends on the radius of the droplet. After two to three oscillation cycles one usually observes a small gas bubble (reaction product $\mathrm{CO}_{2}$ ) that locates itself at the top of the droplet within the BZ/oil interface (see white points at the centers of the larger drops).

showing no oscillations usually exhibited a slow increase in intensity corresponding to a slow oxidation of the catalyst. The inset of Figure $2 \mathrm{a}$ presents the data of the main figure in a plot having a reciprocal $R$-axis. The points scatter to some extent around a line, indicating that the radius-dependent increase in oscillation period follows roughly a $1 / R$ dependence. For drops with $R>7 \mathrm{~mm}$ (not shown), however, we could not resolve any systematic dependence on the radius.

The observed increase in the period of chemical oscillations for decreasing drop radii is caused by a radius-dependent oxygen inhibition. In this context it is important to note that the catalyst concentration is at least 4 times higher than the oxygen concentration in the oil. Consequently, oxygen is consumed completely during each phase of high ferriin concentration. This oxygen consuming excitation is followed by a phase of high bromide and relatively low ferriin concentration, allowing oxygen to accumulate in the droplet via a diffusive influx from the oil. This scenario repeats itself with each oscillation period. The influx of oxygen from the oil is mainly controlled by the surface-to-volume ratio of the droplet that increases for decreasing drop radii. The critical radius of $1.5 \mathrm{~mm}$ below which no oscillations are found could therefore indicate a bifurcation occurring due to an increased influx of oxygen into smaller droplets.

Figure $2 b$ shows a typical period-radius dependence for an experiment carried out under anaerobic reaction conditions. The period of oscillations decreases slightly with decreasing drop radius (maximal change $\approx 10 \%$ ). Even the smallest droplets (radii $\approx 0.1 \mathrm{~mm}$ ) performed chemical oscillations that were easily detected. This finding is in contrast to the existence of a critical radius $(1.5 \mathrm{~mm})$ for aerobic oscillations. In the absence of the inhibitor oxygen, the critical radius is at least 15 times smaller or, most likely, does not exist at all. The small decrease 

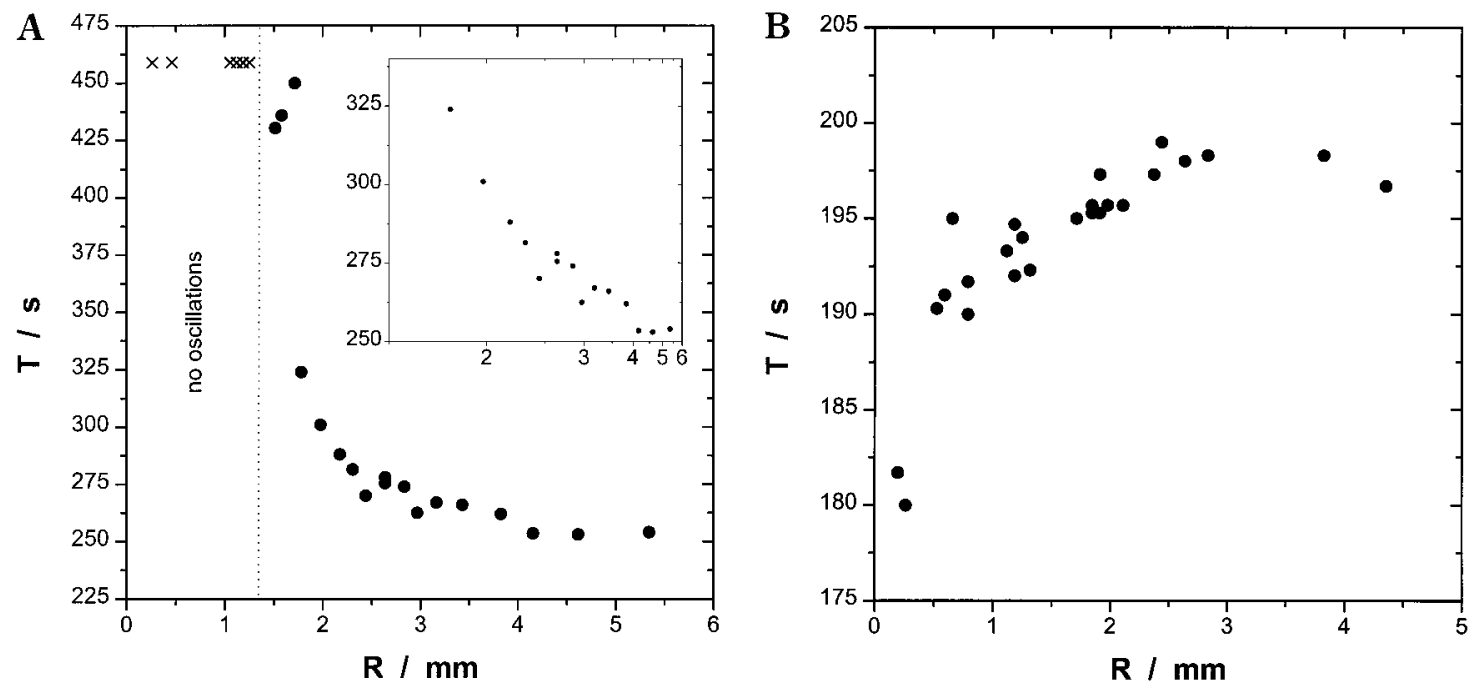

Figure 2. Oscillation period $T$ as a function of the radius $R$ of BZ droplets. (A) Aerobic reaction conditions: The period increases with decreasing radii. Below the critical radius of $R \approx 1.5 \mathrm{~mm}$ no oscillations were observed. The radii of nonoscillatory droplets are indicated by the symbol " $\times$ " at an arbitrary $T$ value; $\left[\mathrm{H}_{2} \mathrm{SO}_{4}\right]=0.1 \mathrm{M}$. The inset shows the results of an aerobic experiment carried out at $\left[\mathrm{H}_{2} \mathrm{SO}_{4}\right]=0.12 \mathrm{M}$ with the $R$-axis having a reciprocal scale. (B) Anaerobic conditions: The period decreases with decreasing radii. No critical radius is found; $\left[\mathrm{H}_{2} \mathrm{SO}_{4}\right]=0.12 \mathrm{M}$.

of oscillation periods observed for small droplets is caused by an diffusive flow of bromine from the BZ drop into the oil. Since the concentrations of bromine and bromide are correlated via the equilibrium (R1), a diffusive loss of $\mathrm{Br}_{2}$ causes a faster decrease of the inhibitory $\mathrm{Br}^{-}$, thus shortening the recovery period. This explanation is supported by additional experiments in which malonic acid was replaced by glyoxylic acid (HCO$\mathrm{COOH}, \mathrm{GOA}){ }^{20}$ GOA and certain other compounds such as oxalic acid ${ }^{21}$ react readily with the oxidized catalyst but cannot undergo bromination. Therefore, the ferroin-catalyzed BZ reaction run with GOA accumulates bromine very rapidly, ferroin is brominated, and a redish-black compound precipitates inside the drops. Experiments with this modified BZ solution showed that only small droplets stayed blue without precipitation. The result demonstrates the efficiency of diffusive bromine removal from drops having a high surface-to-volume ratio. Obviously, the activation of oscillations due to bromine removal is not limited to anaerobic conditions but should also occur in the presence of oxygen.

The spatial phase distribution of oscillations within single droplets shows characteristic patterns that strongly depend on the atmospheric reaction conditions. Homogeneous oscillations were observed if the oil as well as the BZ stock solutions were purged with nitrogen prior to the experiment. For an initially aerobic BZ solution and an oxygen-free oil oscillations started at the boundary of the droplets' bases. From there the excitation spread rapidly toward the center. The underlying phase difference is usually smoothed out within a few oscillation periods, and drops were approaching a state of homogeneous oscillations. It seems likely that these initial phase differences are caused by a diffusive outflow of oxygen from the BZ droplets into the oil phase. Consequently, the inhibition caused by the initial oxygen content of the BZ solution is smaller at the boundary of the drop, thus leading to the observed initial phase shift. Since the first oscillation consumes oxygen completely, the primary source for phase differences has disappeared, and phase diffusion synchronizes the oscillations within the droplet.

In the case of oxygen present in the oil phase, oscillations started in the central region of droplets and spread rapidly outward. Figure 3 shows a typical example of the observed dynamics. The retardation time $\tau(x, y)$ between the excitation at a given point $(x, y)$ and the center of the drop was measured,

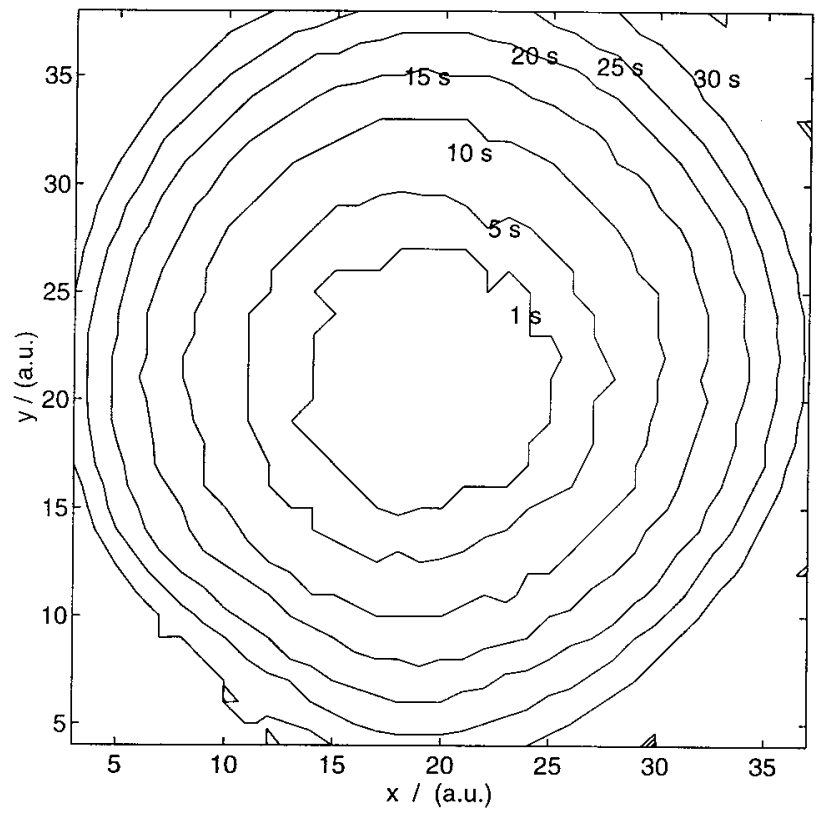

Figure 3. Typical contour plot of a BZ droplet showing curves of constant $\tau$ values, where $\tau(x, y)$ denotes the retardation time between the excitation at the location $(x, y)$ and the center of the droplet. Since the curves are nearly circular and concentric, $\tau$ can be effectively described in polar coordinates: $\tau(r, \phi)=\tau(r)$. Radius of the droplet: $R$ $=3.2 \mathrm{~mm} ;\left[\mathrm{H}_{2} \mathrm{SO}_{4}\right]=0.1 \mathrm{M}$.

by calculating the cross-correlation series $c\left(x, y, \tau^{\prime}\right)$ between the intensity signal $I(x, y)$ and the signal obtained from the center $I_{c}$. The map of retardation times $\tau(x, y)$, shown in Figure 3, is then extracted as the $\tau^{\prime}$ values for which the correlation $c\left(x, y, \tau^{\prime}\right)$ reaches a maximum. Curves of equal excitation time form an array of expanding, concentric circles with their center located in the center of the droplet. The contour plot also reveals that the excitation (i.e., the onset of oscillation) starts to spread rapidly but decelerates while approaching the boundary of the drop. In contrast to the transient phase pattern observed under partially anaerobic conditions, the dynamics exemplified in Figure 3 did not vary within the duration of the experiment ( $>45 \mathrm{~min}$ ). It is, thus, reasonable to assume that the phase gradient is kept constant by a repetitive mechanism involving oxygen consumption and oxygen influx. 

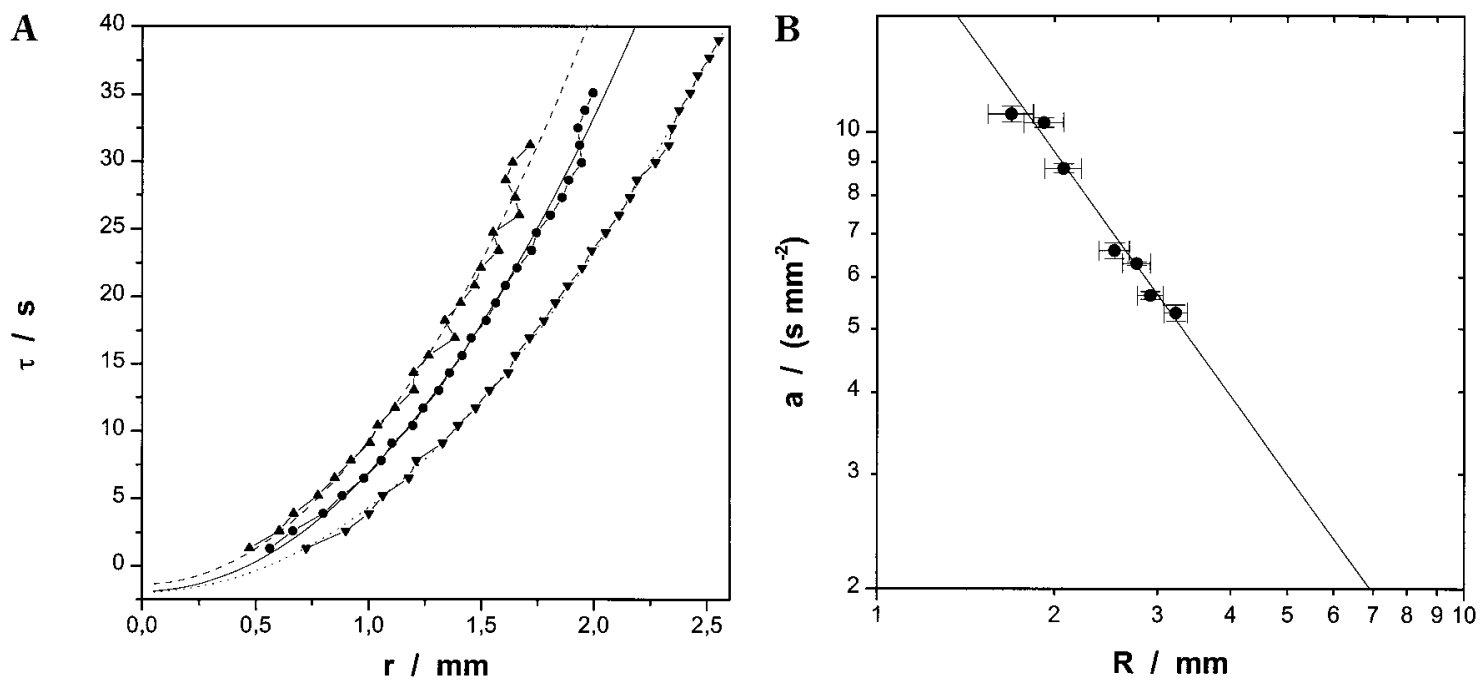

Figure 4. Retardation time $\tau$ as a function of the radial coordinate $r$ for three different BZ droplets: $R=1.69 \mathrm{~mm}(\mathbf{\Delta}), 2.07 \mathrm{~mm}(\bullet), 2.76 \mathrm{~mm}$ $(\boldsymbol{\nabla}) ;\left[\mathrm{H}_{2} \mathrm{SO}_{4}\right]=0.1 \mathrm{M}$. Solid and dashed curves show functions $\tau=a r^{2}+c$ fitted to the experimental data. Values of the parameter $a$ are found to vary with the radius of droplets $R$. (B) Double-logarithmic plot of this dependence within a relatively small range of $R$ values. The solid line shows the result of the linear regression yielding a slope of -1.3 .

Figure $4 \mathrm{a}$ shows the dependence of the retardation time $\tau$ on the radius $r$ for three $\mathrm{BZ}$ droplets having different radii. The data obtained from individual drops were fitted by minimizing the root-mean-square deviations to a simple parabolic function without linear term: $\tau(r)=a r^{2}+c$. Experimental data and parabolic fits are in good agreement. The negative offset $c \approx$ $2 \mathrm{~s}$ is of the order of the sampling time $\Delta t=1.3 \mathrm{~s}$. This deviation from the expected value of zero might also reflect small differences between the measured drop center and the center of curves of equal retardation time. The parameter $a$ determining the steepness of the parabola decreases with increasing drop radius $R$. (Note that $a$ has the unit of an inverse diffusion coefficient.) Figure $4 \mathrm{~b}$ shows a double-logarithmic plot of the dependence $a(R)$. Within the small range of analyzed drop radii, the data indicate a power law dependence of the form

$$
\alpha \propto R^{-s}
$$

The experimental value for $s$ was obtained by linear regression as 1.3. Note that we do not claim that the given value of $s$ is constant over a broad range of drop radii. For drop radii that are relevant for the present investigation, however, the power law (1) and the obtained value of $s$ characterize the experimental data sufficiently well.

\section{Geometrical Consideration}

Liquid drops being in contact with a solid, horizontal surface are subjected to forces arising from surface tension and gravitation. Since their shape cannot be described analytically, ${ }^{22}$ we assume for simplicity the shape to be a spherical (or ellipsoidal) segment and neglect all terms of the order $r^{4}$ or higher. Figure 5 gives a sketch of the droplet and illustrates the definition of some relevant parameters. The height $h$ of the drop surface is given by

$$
h(r)=\left(R_{0}{ }^{2}-r^{2}\right)^{1 / 2}-d ; \quad r \leq R<R_{0}
$$

The BZ droplets studied in the above experiments had a small maximum height $h_{\max }$ and a small height-to-radius ratio $h_{\max } /$ $R$. The oxygen influx from the oil into the oxygen-consuming droplet is expected to cause vertical gradients in the concentra-

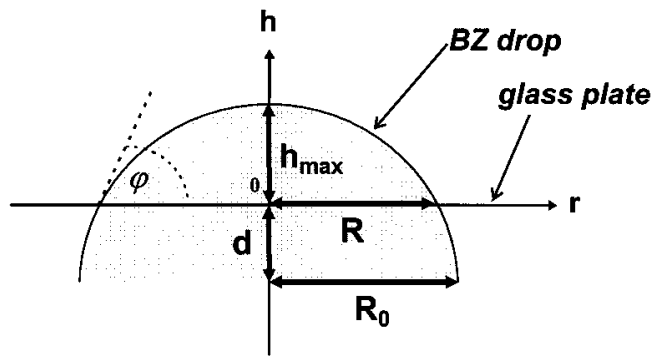

Figure 5. Schematic drawing of an idealized BZ droplet. The droplet is assumed to be spherical with the glass plate cutting the sphere at a height $d$ above the center. $R_{0}$ denotes the radius of the sphere. The definition of $R$ coincides with the one for experimentally measured radii. The height of the droplet is $h_{\max }=R_{0}-d$.

tion of this inhibitor. However, due to the diffusive coupling within the droplets, large phase differences of oscillations in this direction are unlikely and can be neglected in comparison to the maximal phase differences in radial direction. Nevertheless, it is obvious that the vertical oxygen gradient will strongly influence the local recovery time of the system. This effect of oxygen on the onset of oscillations results in a retardation time $\theta$ that we assume to be inversely proportional to the drop height $h$. Based on the Taylor expansion of the expression given in eq $2, \theta$ is approximated by

$$
\theta=\xi / h(r)=\frac{\xi}{R_{0}-d}+\frac{\xi r^{2}}{2 R_{0}\left(R_{0}-d\right)^{2}}+O\left(r^{4}\right)
$$

where $\xi$ denotes a constant parameter accounting for various factors such as the transport properties of oxygen and the oxygen chemistry in the particular BZ system. Notice that the term $\xi /\left(R_{0}-d\right)$ in (3) does not depend on the radial coordinate $r$. Hence, (3) is yielding equations for the oscillation period $T=$ $T_{0}+\theta(r=0)$ and the radius-dependent retardation $\tau(r)=\theta(r)$ $-\theta(r=0)$ within a single droplet:

$$
\begin{aligned}
& T=T_{0}+\frac{\xi}{R_{0}-d} \\
& \tau=\frac{\xi r^{2}}{2 R_{0}\left(R_{0}-d\right)^{2}}
\end{aligned}
$$


where $T_{0}$ is the oscillation period of an infinite $\mathrm{BZ}$ volume. Equation 4a implies a constant period in the droplet which is governed by the oscillations occurring at $r=0$. The strong spatial coupling within the drops, however, might also result in some average contribution from the $r^{2}$ term of eq 3 . Note that eq $4 \mathrm{~b}$ predicts $\tau$ to be zero for $r=0$, which is consistent with the definition of the experimental retardation time $\tau$ introduced above.

Equations $4 \mathrm{a}$ and $4 \mathrm{~b}$ describe the period of oscillations and the retardation time in terms of the parameters $R_{0}$ and $d$. These parameters are related to the experimentally oberved radius $R$ of the drop's base by the equation $R^{2}=R_{0}^{2}-d^{2}$. Since this equation is not sufficient to eliminate $R_{0}$ and $d$ from $(4 \mathrm{a}, \mathrm{b})$, one has to introduce an additional criterion for the shape of the droplets. In the following we consider two limiting cases: (a) The shape of the drop is governed by a constant contact angle $\varphi=\arccos \left(d / R_{0}\right)$ (cf. Figure 5). Consequently, the ratio $\alpha \equiv$ $R_{0} / d$ does not change with the size of the droplet. (b) The shape is ellipsoidal with $\beta \equiv R_{0} / R \equiv$ constant and also limited by a constant height $h_{\max } \equiv R_{0}-d$. Both cases are limits in the sense that the maximal height can neither decrease with increasing $R$ (case b) nor grow faster than $h_{\max } \propto R$ (a). The assumption of an ellipsoidal shape in (b) is not crucial but allows the deduction of a simple power law for $\tau$.

In the limiting case of a constant contact angle (a) eqs $4 a$ and $4 \mathrm{~b}$ yield

$$
\begin{aligned}
& T=T_{0}+\frac{\xi\left(\alpha^{2}-1\right)^{1 / 2}}{\alpha-1} \frac{1}{R} \\
& \tau=\frac{\xi}{2}\left[\frac{(\alpha+1)^{3}}{\alpha^{2}(\alpha-1)}\right]^{1 / 2} \frac{1}{R^{3}} r^{2}
\end{aligned}
$$

The oxygen-induced change in oscillation period is inversely proportional to the radius $R$ of the base of the drop. This result is in good agreement with the experimental findings shown in Figure 1. For the dependence of the retardation time $\tau$ on $R$, one obtains a power law of the form $\tau \propto a \propto R^{-3}$ or, in terms of the earlier notation (1), s=3. Equations $5 \mathrm{a}$ and $5 \mathrm{~b}$ also predict that $T-T_{0}$ and $\tau$ are nontrivial functions of $\alpha$. The fact that $\alpha^{-1}$ denotes the cosine of the contact angle $\varphi$ opens the possibility to compare the above functions with experimental data that could be obtained by changing the material of the Petri dish (here glass). In the framework of the present study, however, this relation has not been investigated.

In the limit of constant drop height $(b)$, the oscillation period $T$ shows no dependence on $R$ :

$$
T=T_{0}+\xi / h_{\max }
$$

a finding that could be valid for larger BZ droplets that were not investigated in detail and for which the assumption of $h_{\max }$ $=$ constant should be a reasonable approximation. For the retardation time one obtains

$$
\tau=\frac{\xi}{2 R_{0} h_{\max }^{2}} r^{2}=\frac{\xi}{2 R \beta h_{\max }^{2}} r^{2}
$$

where the right term is based on the assumption of an ellipsoidal shape of droplets. This expression yields a power law with $s$ $=1$. Note that eqs $5 \mathrm{~b}$ and $6 \mathrm{~b}$ for the dependence of the retardation time $\tau$ on the radial coordinate $r$ have neither a linear nor a cubic contribution, thus explaining the small residuals found between the experimental data on $\tau(r)$ and the corresponding parabolic fits $\tau=a r^{2}+c$ (compare Figure 4a). The presented geometrical analysis yields the values one and three as limits for the exponent $s$ of the power law $a \propto R^{-\mathrm{s}}$. The experimental value of $s=1.3$, obtained from the data shown in Figure 4b, is therefore in agreement with the above considerations.

\section{Discussion}

Molecular oxygen is the key species controlling the radius dependence of oscillation periods and phase gradients within the BZ droplets. Under aerobic conditions the oil layer acts as an effectively infinite reservoir of this inhibitor. Due to the high catalyst concentration used in this study, the amount of organic radicals produced is high enough to consume oxygen completely. While consumption should dominate during the excited state and the early refractory phase of oscillations, oxygen can accumulate during the phases of low ferriin concentrations. Hence, successive excitations give rise to bromide levels that are above the bromide concentrations reached under anaerobic conditions, and the period of oscillations is increased. The concentration of oxygen diffusing into the droplet changes with distance from the droplet's center. The resulting phase wave $^{23}$ is nontransient, because the driving chemical gradient is renewed with each oscillation cycle. This behavior differs completely from the dynamics of phase waves in two-dimensional oscillatory reaction-diffusion systems, where initial phase gradients disappear rapidly due to diffusive coupling of the local oscillators. ${ }^{23}$ An additional effect arises from the diffusive flow of bromine from the aqueous BZ solution into the oil phase. Since bromine and bromide are coupled by the equilibrium (R1), one observes a radiusdependent, weak activation of oscillations. Note that the diffusion of ionic (e.g., $\mathrm{Br}^{-}$) or other hydrophilic BZ species (e.g., $\mathrm{HBrO}_{2}$ ) into the oil phase is unlikely.

The surface-to-volume ratio of a spherical system decreases inversely proportional to its radius and is, therefore, an important parameter controlling the efficiency of the diffusive flux between the system and its surroundings. Based on this general principle, a simple geometrical analysis for the radius-dependent inhibition of flat BZ droplets was derived. Despite major simplifications, the analysis elucidates several of the observed phenomena, such as (a) the rough $1 / R$ dependence of the change in oscillation periods, (b) the good agreement between experimental data on the phase retardation $\tau$ on the radial coordinate $r$ and parabolic fits $\left(\tau=a r^{2}\right)$, and (c) the power law $a \propto R^{-1.3}$ found for the range of investigated radii.

The geometrical analysis yields only a qualitative understanding of the experimental data. One of its central assumptions is that the oxygen gradient in the vertical direction is sufficiently steep to generate a homogeneous response of the BZ system. While this assumption is justified by experimental observations, quantitative details of the relation between oscillations in systems with steep oxygen gradients and systems with homogeneous oxygen concentrations remain unclear. We therefore see a need for new reaction-diffusion models that are capable of describing the dynamics of oxygen inhibition as well as the consumption of this important inhibitor. The presented results should be an interesting test case for these kinds of models. Finally, we like to stress that other experimental observations, such as effects of oxygen on excitation wave propagation ${ }^{24}$ or the stratification of thin-layered BZ systems which gives rise to so-called crossing waves, ${ }^{11,18}$ are also strongly related to the interaction of oxygen transport and consumption. 
Acknowledgment. We thank B. Neumann, H. Sevcikova, U. Storb, and V. S. Zykov for fruitful discussions. This research was supported by the Fonds der Chemischen Industrie.

\section{References and Notes}

(1) Thompson, D'Arcy W. In On Growth and Form; Bonner, J. T. Ed.; Cambridge University Press: Cambridge 1961.

(2) Schnepf, E. Bau der Zelle. In Biophysik; Hoppe, W., Lohmann,

W., Markl, H.; Ziegler, H., Eds.; Springer: Berlin, 1982.

(3) Field, R. J., Burger, M., Eds. Oscillations and Traveling Waves in Chemical Systems; Wiley-Interscience: New York, 1985.

(4) Murray, J. D. Mathematical Biology; Springer: Berlin, 1989. 8649 .

, R. J.; Körös, E.; Noyes, R. M. J. Am. Chem. Soc. 1972, 94

(6) Maselko, J.; Swinney, H. L. J. Chem. Phys. 1986, 85, 6430. Wang, J.; Hynne, F.; Sørensen, P. G. Int. J. Bifurcat. Chaos. 1996, 6, 1267.

(7) Zaikin, A. N.; Zhabotinsky, A. M. Nature 1970, 225, 535. Vinson, M.; Mironov, S.; Mulvey, S.; Pertsov, A. Nature 1997, 386, 477. Tyson, J. J.; Keener, J. P. Physica 1988, D32, 327.

(8) Lechleiter, J.; Girard, S.; Peralta; E.; Clapham, D. Science 1991, 252,123 .

(9) Siegert, F.; Weijer, C. J. Physica 1991, D49, 224. Tomchik, K. J. Devreotes, P. N. Science 1981, 212, 443.

(10) Scott, S. K. Oscillations, Waves and Chaos in Chemical Kinetics Oxford University Press: Oxford, 1994.

(11) Zhabotinsky, A. M.; Müller, S. C.; Hess, B. Chem. Phys. Lett. 1990, 172, 445 .

(12) Roux, J.-C.; Rossi, A. C. R. Hebd. Sceances Acad. Sci., Ser. C 1978, 287, 151

(13) Barkin, S.; Bixon, M.; Noyes, R. M.; Bar Eli, K. Int. J. Chem. Kinet. 1978, 10, 619. Brusa, M. A.; Perissinotti, L. J.; Coloussi, A. J. J. Phys. Chem. 1985, 89, 1572. Försterling, H.-D.; Noszticzius, Z. J. Phys. Chem. 1989, 93, 2740.

(14) Neumann, B.; Müller, S. C.; Hauser, M. J. B.; Steinbock, O.; Simoyi, R. H.; Dalal, N. S. J. Am. Chem. Soc. 1995, $117,6372$.
(15) Krug, H.-J.; Pohlmann, L.; Kuhnert, L. J. Phys. Chem. 1990, 94 , 4862

(16) Kuhnert, L.; Pohlmann, L.; Krug, H.-J. Physica 1988, D29, 416. Showalter, K.; Noyes, R. M.; Turner, H. J. Am. Chem. Soc. 1979, 101 , 7463.

(17) For anaerobic conditions (R5, R6) imply a stoichiometry [Ce(IV) $]_{\text {consumed }} /\left[\mathrm{Br}^{-}\right]_{\text {produced }}$ of two, which is in agreement with experimental findings: Försterling, H.-D.; Stuk, L.; Barr, A.; McCormick, W. D. J. Phys. Chem. 1993, 97, 2623. Note that the stoichiometry changes to one for aerobic reaction conditions: Jwo, J. J.; Noyes, R. M. J. Am. Chem. Soc. 1975, 97, 7320.

(18) Zhabotinsky, A. M.; Györgyi, L.; Dolnik, M.; Epstein, I. R. J. Phys. Chem. 1994, 98, 7981.

(19) Yoshikawa et al. (Yoshikawa, K.; Kusumi, T.; Ukitsu, M.; Nakata, S. Chem. Phys. Lett. 1993, 211, 211) report periodic changes in surface tension for oscillating homogeneous BZ systems. Hence, one could expect periodically changing contact angles and drop shapes. In the present study, however, pinning of the contact line suppressed this effect. It will be interesting to see whether BZ droplets on other surfaces (e.g., Teflon) might allow a systematic study of this chemomechanical phenomenon.

(20) Neumann, B.; Steinbock, O.; Müller, S. C.; Dalal, N. S. J. Phys. Chem. 1996, 100, 12342 .

(21) Oscillations in the BZ reaction with oxalic acid substrate have been observed if the produced bromine is removed by an inert carrier gas: Noszticzius, Z. Stirling, P.; Witmann, M. J. Phys. Chem. 1985, 89, 4914.

(22) The underlying differential equation can be derived from the Laplace equation $\sigma / r_{1}+\sigma / r_{2}=g \rho z+C$, where $r_{1}, r_{2}, g, \rho, z$, and $C$ denote the radii of mean curvature, gravitational acceleration, density of the liquid, vertical height, and a constant, respectively: See e.g.: Bergmann-Schaefer Lehrbuch der Experimentalphysik; Walter de Gruyter: Berlin, 1974; Vol. I.

(23) Ross, J.; Müller, S. C.; Vidal, C. Science 1988, 240, 460. Notice that phase waves can undergo a transition to diffusion-driven excitation waves: Reusser, E. J.; Field, R. J. J. Am. Chem. Soc. 1979, 101, 1063.

(24) Taylor, A. F.; Johnson, B. R.; Scott, S. K. J. Chem. Soc., Faraday Trans. 1998, 94, 1029. 